\title{
Introduction to the special issue on TAISA 2007
}

\author{
Ian O'Connor
}

Published online: 2 July 2010

(C) Springer Science+Business Media, LLC 2010

This special issue of Analog Integrated Circuits and Signal Processing is dedicated to selected best papers taken from the TAISA Conference (Analog Information and Signal Processing and its Applications) held in Lyon in October 2007. The full-length papers describe the selected works in a format extended from the conference proceedings. Six best papers were selected for publication in this issue by the technical program committee from all the contributions at the conference and were subject to the normal journal review and refereeing process. The six papers include two papers on data conversion, one on radiofrequency circuits, two on image sensors and one on noise analysis.

The first paper, by H.A. Khushk et al., presents a novel architecture for high-pass cascaded $\Delta \Sigma$ modulators, to be used in applications sensitive to low-frequency noise. With respect to conventional cascade high-pass $\Delta \Sigma$ modulator architectures, this work attains better dynamic range, stability and achievable SNR, and demonstrates additional flexibility in the use of any second-order modulator architecture in the first stage. In the second paper, S. Guessab et al. propose ways to enhance the linearity and resolution of analog-to-digital converters with three mixed (analog and digital) loop architectures. Mathematical models and high-level simulation show the benefits of the topologies and evaluate the impact of common non-idealities. One of the loop topologies is in particular able to enhance resolution by 5 bits without compromising bandwidth, and with only a minor increase in complexity. The most promising ideas have been included in a prototype realized in a $0.35 \mu \mathrm{m}$ CMOS technology.

I. O'Connor ( $\square)$

Lyon Institute of Nanotechnology, Ecole Centrale de Lyon,

Lyon, France

e-mail: ian.oconnor@ec-lyon.fr
M. Battista et al. present the design and implementation of an Ultra-WideBand (UWB) CMOS LC filter LNA for carrier-less impulse radio receivers in the third paper. Both single- and differential-ended LNA architectures are proposed for the two main variants of fractional bandwidths (ECC and FCC frequency bands). Throughout the paper, design and layout guidelines are given for performance and reliability optimization, and are demonstrated with test results for several LNA prototypes in a $0.13 \mu \mathrm{m}$ CMOS technology.

In the fourth paper, D. Ginhac et al. present a high-speed programmable analog VLSI image acquisition and lowlevel image processing system on chip. The architecture of the chip is based on a dynamically reconfigurable singleinstruction multiple data (SIMD) processor array. Hence the architecture is massively parallel and enables the computation of programmable image processing in each pixel. A $64 \times 64$ pixel proof-of-concept chip was fabricated in a $0.35 \mu \mathrm{m}$ standard CMOS process, with a pixel size of $35 \mu \mathrm{m} \times 35 \mu \mathrm{m}$. The chip can capture raw images up to $10 \mathrm{kfps}$ and can run low-level image processing at a frame-rate of 2-5 kfps. O. Bulteel et al. present a fully integrated blue/ultra-violet $(\lambda=200-450 \mathrm{~nm})$ SOI CMOS photosensor in the fifth paper for biomedical and environmental applications. The pixel includes a photodiode and the analog processing circuit of the photodiode signal, fully integrated in a $2 \mu \mathrm{m}$ SOI CMOS technology, and is shown experimentally to demonstrate good linear behavior, sensitivity and efficiency.

Finally in the sixth paper, P. Pittet et al. propose a cyclostationarity-based model for noise analysis of charge amplification with correlated double sampling (CDS). Through this model, it is shown how CDS operation has various impact on noise: reset noise is suppressed, 1/f noise is attenuated, but there is no effect on the input referred 
noise current. This model is validated by comparison of analysis results with those from temporal noise analysis using conventional electrical simulation. Evaluation speedup is shown to be significant.

As guest editor of this special issue, I would like to thank all the presenters at the conference from these sessions for having undertaken the extensions to the original conference paper, the technical program committee for the selection of the best papers, as well as the anonymous reviewers for their feedback and help in ensuring that the papers met the required level of quality. I also wish to extend my appreciation to the main editor of ALOG, Prof. M. Ismail, for his guidance, and finally S. Shenbagam for putting this issue together.

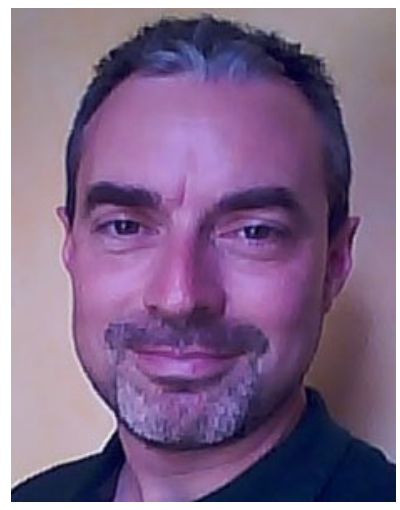

Ian O'Connor (IEEE S'95M'98-SM'07, IEE S'87-M'98) is Professor for Heterogeneous and Nanoelectronics Systems Design in the Department of Electronic, Electrical and Control Engineering at Ecole Centrale de Lyon, France. He is currently head of the Heterogeneous Systems Design group at the Lyon Institute of Nanotechnology, of which he is also one of the vice-directors. Since 2008, he also holds a position of Adjunct Professor at Ecole Polytechnique de Montréal, Canada. His research interests include design methods and tools for physically heterogeneous systems on chip, and their application to novel system architectures based on non-conventional devices. He has authored or co-authored around 100 book chapters, journal publications and conference papers and has been workpackage leader or scientific coordinator for several national and European projects. He also serves as an expert with the french Observatory for Micro and Nano Technologies (OMNT) and with IFIP WG10.5. 